\title{
PROPOSTA DE SISTEMATIZAÇÃO DOS REQUISITOS DE SUSTENTABILIDADE PARA AUXILIAR O DESENVOLVIMENTO DE PROJETOS DE EDIFÍCIOS ${ }^{1}$
}

\author{
PROPOSAL FOR SYSTEMATIZATION OF REQUIREMENTS FOR \\ SUSTAINABILITY TO ASSIST THE DESIGN OF BUILDING DEVELOPMENT
}

\author{
Daniel Reis \\ Universidade de São Paulo (USP) \\ danielreis@usp.br \\ Márcio Fabrício \\ Universidade de São Paulo (USP) \\ marcio@sc.usp.br \\ Lucas Melchiori \\ Universidade de São Paulo (USP) \\ Ic.melchiori@gmail.com \\ José Sousa \\ Instituto Superior de Engenharia do Porto (ISEP) \\ iso@isep.ipp.pt
}

\begin{abstract}
Resumo
A indústria da construção é um dos maiores e mais ativos setores da economia mundial. No entanto, apresenta impactos negativos ao longo do ciclo de vida dos edifícios, colocando em causa os desígnios do desenvolvimento sustentável que procura um equilíbrio entre as vertentes ambiental, econômica e social. Por outro lado, a fase de projeto tem-se revelado crucial para que o edifício consiga atingir níveis elevados de sustentabilidade uma vez que as decisões tomadas nesta fase influenciam de forma significativa o desempenho da construção. O objetivo deste trabalho consiste em apresentar uma ferramenta que permita auxiliar os projetistas durante 0 desenvolvimento de projetos de edifícios. Foi elaborada recorrendo a um conjunto de processos que se encontram estruturados em função das várias fases do ciclo de evolução de um projeto e das várias especialidades do mesmo. O desenvolvimento dos vários processos formam uma sequência de estados que proporciona aos diversos intervenientes do projeto uma estrutura básica para a sua elaboração. Por meio da correta aplicação desta ferramenta será possível uma melhor coordenação entre a equipe projetista ao longo do desenvolvimento do projeto e resultará num maior equilíbrio da construção ao nível das três vertentes do desenvolvimento sustentável.
\end{abstract}

Palavras-chave: Gestão de projetos. Melhores práticas de projeto. Sustentabilidade na construção.

\section{Abstract}

The construction industry is one of the largest and most active sectors of the world economy. However, it has negative impacts throughout the life cycle of buildings, putting in question the intentions of sustainable development, which seeks a balance between environmental, economic and social aspects. Moreover, the design phase has

${ }^{1}$ REIS, D.; FABRÍCIO, M.; MELCHIORI, L.; SOUSA, J. Proposta de sistematização dos requisitos de sustentabilidade para auxiliar o desenvolvimento de projetos de edifícios. In: ENCONTRO BRASILEIRO DE TECNOLOGIA DE INFORMAÇÃO E COMUNICAÇÃO NA CONSTRUÇÃO, 7., 2015, Recife. Anais... Porto Alegre: ANTAC, 2015. 
proven to be crucial for building achieve high levels of sustainability since the decisions taken at this stage significantly influence the performance of the construction. The objective of this study is to provide an instrument to help designers during the development of building projects. It has been developed using a set of processes that are structured according to the various stages of evolution of a project cycle and the various specialties of it. The development of the various processes form a sequence of states that provides to the various project stakeholders a basic framework for its elaboration. Through the proper application of this tool it will be possible to achieve a better coordination between the design team throughout the project development and will result in a better balance of the building in terms of the three aspects of sustainable development.

Keywords: Design management. Better design practices. Sustainable construction.

\section{INTRODUÇÃO}

A indústria da construção representa um dos maiores contributos na economia global, mas também impactos negativos ao longo do ciclo de vida dos edifícios evidenciando a necessidade de promover e adotar mudanças na forma como se concebem e constroem os edifícios (ZUO; ZHAO, 2014). Para responder a estas exigências são necessárias ferramentas capazes de avaliar o nível de sustentabilidade de cada edifício, permitindo uma comparação entre eles e a prática comum, evidenciando aqueles que têm comportamento quantitativo superior aos demais (FERREIRA et al., 2014). Neste contexto, ao longo das últimas duas décadas têm surgido um número significativo destas ferramentas. A nível internacional destacam-se o Building Research Establishment's Environmental Assessment Method (BREEAM), o Sustainable Building Tool (SBTool) e o Leadership in Energy and Environmental Design (LEED) (internacionais); no Brasil destacam-se O AQUA e o LEED Brasil (CASTANHEIRA; BRAGANÇA, 2014) (KIBERT, 2013). Estes sistemas baseiam-se numa ponderação entre diversas categorias e indicadores que, no final, resultarão na classificação da sustentabilidade do edifício. Existem também ferramentas baseadas em Análise de Ciclo de Vida (ACV) que permite quantificar o potencial de impacto ambiental de produtos ou processos durante todo o seu ciclo de vida. Ainda sobre este aspeto, a União Europeia por meio do Comité Técnico CEN/TC 350 tem vindo a desenvolver uma série de normas para avaliar a sustentabilidade na construção (EN15643-1, 2010), abordando aspetos ambientais (EN15643-2, 2011) (EN15978, 2011), aspetos sociais (EN15643-3, 2012) e aspetos econômicos (EN15643-4, 2012).

Os edifícios são produtos complexos, envolvem diversos materiais e sistemas construtivos com características bastante distintas e todo o processo de construção incide em diversas áreas de conhecimento. A multidisciplinaridade associada aos edifícios exige que todos aqueles que participam no ato de conceber, construir e utilizar os edifícios se reúnam desde o início da concepção do projeto de modo a que seja possível orientar a tomada de decisão ao longo das várias fases de evolução do projeto. Na realidade, a construção sustentável requer a criação de uma equipe de projeto envolvendo todas as partes interessadas (clientes, projetistas, empreiteiro e consultores) desde a fase embrionário do projeto. Esta abordagem é também conhecida por Processo Integrado de Projeto (IDP)2. A abordagem IPD apresenta responsabilidades adicionais para a equipa projetista, bem como um nível de comunicação mais elevado entre os vários intervenientes envolvidos na elaboração do projeto (DEANE, 2008). Segundo Magent et al. (2009), o desenvolvimento de projetos de edifícios sustentáveis, usualmente requerem uma abordagem integrada e de cooperação entre as várias especialidades, tais como a arquitetura, estruturas, equipamentos e instalações mecânicas e elétricas, equipamentos e instalações de água e esgotos e gestão de resíduos.

Neste sentido, o presente trabalho procurou desenvolver uma ferramenta inovadora e de simples aplicação que permitisse auxiliar os principais intervenientes das diversas especialidades que constituem o processo de projeto (arquitetura, estruturas, instalações mecânicas e hidráulicas) na definição dos passos a dar para incorporar soluções, tecnologias

${ }^{2}$ Acrônimo da expressão da língua inglesa "integrated design process" 
e métodos construtivos com requisitos de sustentabilidade ao longo das várias fases de desenvolvimento do projeto. A ferramenta foi desenvolvida recorrendo a uma pesquisa bibliográfica com recolha de informação que, posteriormente, permitiu a elaboração de um conjunto de processos que se encontram estruturados em função das várias fases do ciclo de evolução de um projeto e das várias especialidades do mesmo. Admite-se que por meio da correta utilização desta ferramenta, será possível uma maior eficiência na comunicação entre os vários intervenientes do projeto e também uma maior eficácia na incorporação de informação técnica com requisitos de sustentabilidade ao longo do ciclo de evolução de um projeto.

\section{SISTEMAS DE AVALIAÇÃO DA SUSTENTABILIDADE DE EDIFÍCIOS}

No decorrer da procura pela sustentabilidade na construção, uma das definições mais consensuais de construção sustentável foi apresentada por Charles Kibert em 1994, no Conselho Internacional da Construção (CIB), que refere o seguinte: "Construção sustentável é a criação e gestão responsável de um ambiente construído saudável, tendo em consideração os princípios ecológicos e a utilização eficiente dos recursos". Posteriormente, e com base nesta definição, o CIB definiu sete princípios para a construção sustentável: i) reduzir o consumo de recursos; ii) reutilizar recursos; iii) utilizar recursos recicláveis; iv) proteger a natureza; v) eliminar produtos tóxicos; vi) analisar os custos de ciclo de vida; vii) assegurar a qualidade.

Estes sete princípios devem ser aplicados em todo o ciclo de vida de um edifício, desde a fase mais preliminar de projeto até ao tratamento dos produtos da demolição/desconstrução (Mateus, 2009). Para responder a estas exigências, têm vindo a ser desenvolvidos, um pouco por todo o mundo, um conjunto significativo de sistemas de avaliação da sustentabilidade de edifícios. Estes sistemas têm como objetivo avaliar quantitativamente os aspetos da sustentabilidade dos edifícios, recorrendo a um conjunto de indicadores de diversas categorias e comunicar os resultados decorrentes da avaliação através de um relatório de fácil interpretação, mesmo quando consultado por pessoas não especializadas na área (BRAGANÇA, et al., 2010) (COLE, 1999). Contudo, apesar de existir diversos sistemas de avaliação de sustentabilidade, cada um com as suas valências distintas, atualmente ainda não existe um sistema que seja internacionalmente aceito. Este fenômeno ocorre devido à subjetividade com que é interpretado o conceito "construção sustentável", motivada principalmente pelas diferenças políticas, tecnológicas, culturais, sociais e econômicas, existentes não só entre países, mas também, dentro de cada país, entre cada região. Mais ainda, o conceito "sustentável" não imutável ao longo do tempo, dependendo igualmente do estado de desenvolvimento científico e tecnológico em cada momento (MATEUS, 2009).

\section{IMPORTÂNCIA DO PROJETO PARA A CONSTRUÇÃO SUSTENTÁVEL}

A importância de considerar a sustentabilidade logo no início do desenvolvimento do projeto atende à necessidade de encontrar soluções de longo prazo que garantam um equilíbrio entre as três dimensões do desenvolvimento sustentável (ambiental, social e econômica). Vários autores reconhecem a importância da fase de projeto para se atingirem níveis de sustentabilidade superiores à prática. Na realidade, é durante a fase de projeto que são definidos a maioria dos materiais e métodos de construção, bem como são determinadas as questões relacionadas com a funcionalidade e qualidade do edifício (REKOLA et al., 2012). Adicionalmente, se o projeto for bem planejado desde o seu início, incluindo os critérios de sustentabilidade logo nesta fase inicial, a possibilidade de reduzir os impactos negativos é maior, bem como o custo de implementação será menor (KOHLER; MOFFATT, 2003).

Por outro lado, os projetos de edifícios sustentáveis usualmente requerem uma abordagem integrada que oriente a tomada de decisões no que se refere ao consumo de recursos (água, 
energia e materiais), qualidade do ar interior, conforto dos utilizadores e potencial de reciclagem e reutilização dos Resíduos da Construção e Demolição (RCD) (MANENT et al., 2009). Este tipo de abordagem apresenta responsabilidades adicionais para a equipe projetista (em comparação com o modelo tradicional de projeto ${ }^{3}$ ), bem como um nível de comunicação mais elevado entre os mesmos (KIBERT, 2013). Efetivamente o desenvolvimento de um projeto sustentável é mais facilmente atingido por meio de uma abordagem integrada e orientada para os principais intervenientes do projeto que participam nas fases mais preliminares de projeto, de modo a permitir que estes durante o processo de IPD, tenham a possibilidade de utilizar os seus conhecimentos para influenciar as primeiras decisões de projeto.

Por outro lado, um dos maiores entraves à coordenação dos projetos reside na falta de troca de informação entre os diversos técnicos projetistas. Estes, muitas vezes atuam de forma isolada e sem qualquer troca de informação com os restantes (PEREIRA, 2014). A troca de informação que se afigura necessária não se deve processar unicamente na vertical, devendo ser partilhada numa ótica que envolva a sua transmissão na horizontal (entre especialidades). Ainda segundo o mesmo autor, deverá existir aplicações informáticas específicas que promova e auxilie a gestão e a troca de informação entre especialidades de modo a garantir que se atenda progressivamente aos objetivos e especificações das partes interessadas. A ferramenta apresentada neste artigo procura também ser um contributo à sistematização dos conceitos, da sua integração e da sequenciação da sua implantação na gestão integrada de um projeto de construção.

Atualmente, um dos principais desafios passa por desenvolver e implementar uma metodologia sistemática que sirva de suporte ao processo de concepção de um edifício. Esta metodologia deve contribuir para um equilíbrio mais adequado entre as diferentes dimensões de sustentabilidade, sendo ao mesmo tempo, prático, transparente e suficientemente flexível (BRAGANÇA, et al., 2010). Deste modo, o presente trabalho procurou desenvolver uma ferramenta permita efetuar uma abordagem integrada às diferentes especialidades que constituem o processo de projeto, nas suas diferentes fases de evolução, conjugando as necessidades de cada uma delas com os requisitos de sustentabilidade do sistema LEED-NC e de alguns normativos nacionais e internacionais, que se façam pertinentes.

No capítulo seguinte detalhar-se-á a forma como foi elaborada a ferramenta e como se encontra organizada.

\section{FERRAMENTA PROPOSTA}

A ferramenta que este trabalho se propõe apresentar tem como objetivo o desenvolvimento de um conjunto de processos que permita auxiliar as principais especialidades de projeto a experimentar soluções de sustentabilidade na elaboração de projetos de edifícios. Os processos encontram-se estruturados em função das várias fases do ciclo de evolução de um projeto e das várias especialidades do mesmo. Admite-se que a forma como a ferramenta foi estruturada permite que se criem sinergias entre os vários membros da equipe de projeto, por meio de uma comunicação mais eficiente entre as várias especialidades, bem como a disponibilização de informação técnica necessária para que estes tenham a possibilidade de influenciar o desempenho do edifício desde a fase inicial de projeto até à sua conclusão.

Os requisitos de sustentabilidade que os compõem os foram obtidos por meio de algumas normas brasileiras ABNT, incluindo a norma de desempenho NBR 15.575:2013; algumas

\footnotetext{
${ }^{3}$ Considera-se como modelo tradicional a metodologia conceção-concurso-construção, do inglês design-bid- build. O cliente contrata um arquiteto para desenvolver um programa e de seguida realiza a conceção do projeto com auxílio dos engenheiros. Posteriormente existem propostas por parte de empreiteiros para licitar a obra e o escolhido constrói a obra.
} 
Especificações Técnicas Europeias; e do sistema de certificação de sustentabilidade LEEDNC. Cada processo encontra-se estruturado da seguinte forma: informações de referência a utilizar; informações técnicas a produzir; e documentos técnicos a apresentar.

O desenvolvimento dos vários processos foram uma sequência de estados que proporciona aos diversos intervenientes do projeto uma estrutura básica para a sua elaboração, independentemente do tamanho e complexidade que este possa apresentar.

A opção de utilização do sistema LEED-NC resulta no facto de ser um dos sistemas mais usados no Brasil e também internacionalmente. É importante salientar, que a ferramenta parte do pressuposto que a inserção em projeto de medidas e soluções de sustentabilidade expressas nos sistemas LEED corresponde a um ganho do edifício no ponto de vista da sustentabilidade, mesmo tendo em consideração que atualmente não existe uma metodologia que seja internacionalmente aceite.

Por sua vez, e no que se refere ao faseamento do projeto, de acordo com a norma brasileira NBR 13.531/1995, não foram incluídas na ferramenta as etapas iniciais da norma, nomeadamente o levantamento, o programa de necessidades e o estudo de viabilidade. Admite-se que as decisões mais relevantes em termos de sustentabilidade, nomeadamente o tipo de produto, características e nível de desempenho ocorram nas seguintes fases: i) estudo preliminar; ii) anteprojeto; iii) projeto legal; iv) projeto de execução. Desta forma a ferramenta incide apenas nestas quatro etapas. A figura 1 representa as várias fases de desenvolvimento de um projeto de acordo com a norma brasileira ABNT NBR 13.531/1995.

Figura 1 - Fases no desenvolvimento de um projeto

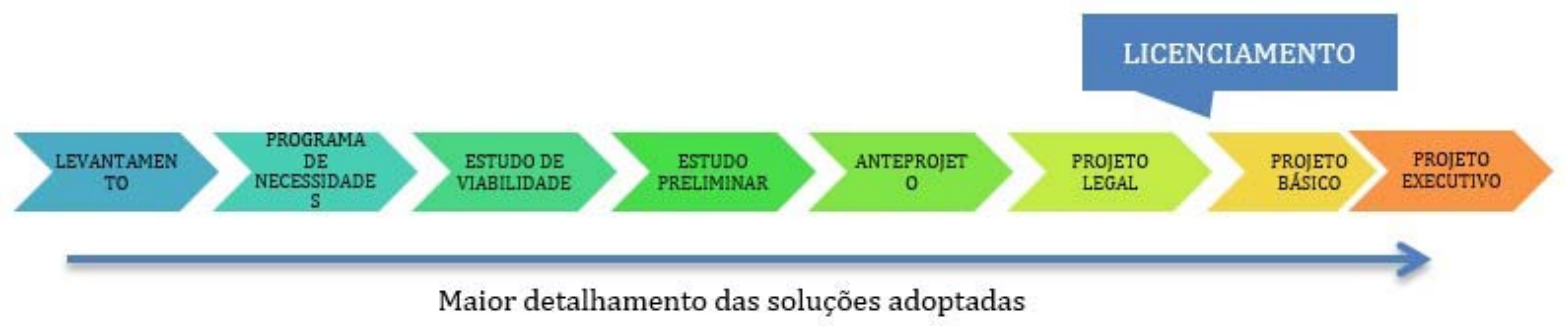

Fonte: Adaptado pelos autores de ABNT NBR 13.531,1995.

Pretende-se que os processos sejam simultaneamente práticos e transparentes para que possam ser utilizados no desenvolvimento de projetos de edifícios de habitação.

Para melhor situar a compreensão do leitor sobre o desenvolvimento da ferramenta, é importante considerar os seguintes aspetos (já abordados no estado de arte):

- Os sistemas de avaliação de sustentabilidade de edifícios podem desempenhar um papel importante na procura da sustentabilidade (DING, 2008) (FERREIRA, et al., 2013).

- Os parâmetros e critérios definidos nestes sistemas devem ser levados em consideração logo na fase inicial de concepção de projeto, permitindo uma colaboração precoce entre as equipas de projeto e as questões de sustentabilidade que se pretendem alcançar (DING, 2008).

De acordo com estes desideratos, os processos desenvolvidos neste trabalho pretendem ser um instrumento facilitador para os principais intervenientes de projeto, permitindo não só disponibilizar informação técnica para auxiliar a tomada de decisão ao longo das várias etapas de desenvolvimento de um projeto, mas também, melhorar a comunicação e o fluxo de informação entre as várias especialidades de projeto. 


\subsection{Organização da ferramenta}

Como se evidenciou, o desenvolvimento da ferramenta baseia-se num conjunto de processos que visa auxiliar os projetistas na definição de soluções, tecnologias e métodos construtivos com requisitos de sustentabilidade ao longo do ciclo de vida de evolução do projeto.

Os processos foram elaborados por meio das folhas de cálculo do "MS-Excel"; foi desenvolvido um por folha. Os vários processos encontram-se interligados entre eles de maneira a aumentar e melhorar não só a comunicação entre os projetistas, mas também a forma como estes se relacionam. Permite-se assim que, por meio de partilha de ideias e opiniões se criem sinergias entre os projetistas, possibilitando incorporar no projeto soluções construtivas visando integrar as necessidades das várias partes interessadas. Por conseguinte, há medida que o projeto evolui o nível de detalhe das soluções construtivas vai aumentando gradualmente abrangendo todos os créditos e pré-requisitos do sistema LEED$\mathrm{NC}$, bem como os documentos normativos que se façam pertinentes. A Figura 2 representa a forma como os processos estão relacionados entre si, em função das várias especialidades de projetos e das várias fases do mesmo. Ao todo foram desenvolvidos 17 processos.

Figura 2 - Representação do mapa de processos

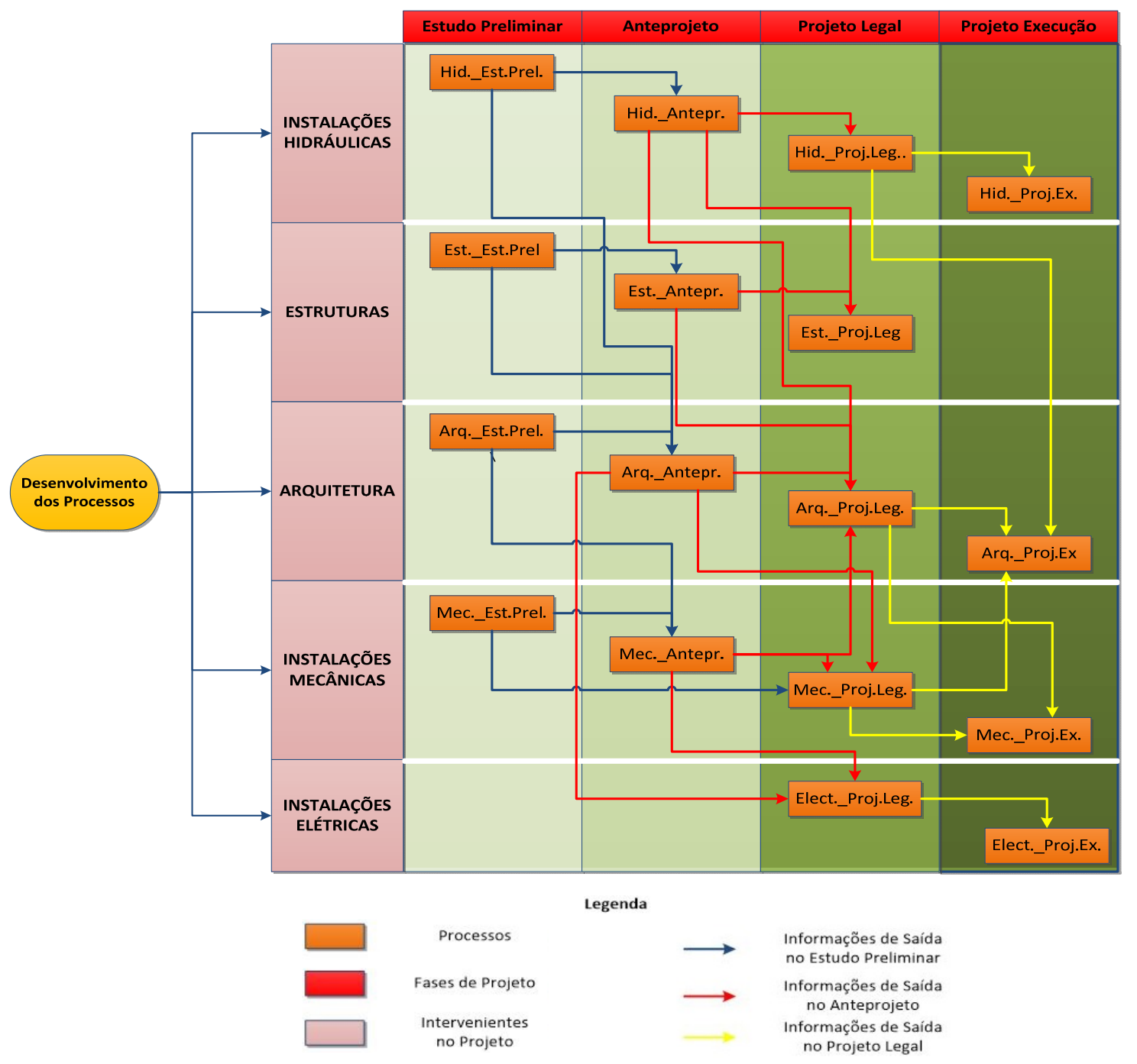

Fonte: Produção dos autores. 
Com efeito, e de acordo com a Figura 2, o mapa de processos segue uma sequência lógica começando logo no estudo preliminar, que orienta as fases subsequentes do projeto por meio de documentos técnicos específicos. Em cada fase do projeto estão previstos diferentes processos em função de cada interveniente, ou seja, no Estudo Preliminar o processo na especialidade de arquitetura é necessariamente diferente da especialidade de estruturas que, por sua vez, também é diferente da especialidade de instalações mecânicas. Permite-se assim que para cada fase de projeto estejam previstos diferentes processos, originando tipos de informação distintos em função de cada especialidade. Considerando o número limitado de páginas para elaboração deste artigo, opta-se por descrever como está organizado apenas um dos processos. Por conseguinte, detalhar-se-á o processo Hid._Antepr. que corresponde à especialidade de Hidráulica na fase de Anteprojeto. Os restantes 16 processos foram desenvolvidos de forma análoga, considerando os diferentes tipos de conhecimento técnico necessário para cumprir com os objetivos de cada fase de projeto em função de cada especialidade. Cada processo é único e a informação técnica que o compõe não é repetitiva.

Segundo a ISO 9000 define processo como "conjunto de atividades inter-relacionadas ou iterativas que transforma entradas em saídas". Com base nesta definição os processos introduzidos na ferramenta foram estruturados contendo "informações de referência a utilizar" (Figura 3) que corresponde às entradas; "informações técnicas a produzir" (Figura 4) que corresponde às atividades a realizar; e por fim "documentos técnicos a apresentar" (Figura 5) que correspondem às saídas.

Figura 3 - Informações de referência a utilizar na especialidade de hidráulica na fase de Anteprojeto

\begin{tabular}{|l|l|}
\hline \multicolumn{1}{|c|}{ Entradas/Informações de referência a utilizar } & \multicolumn{1}{|c|}{ ID Origem } \\
\hline $\begin{array}{l}\text { WEp1/WEc2/WEc3: Informações técnicas relativamente às estratégias que irão ser introduzidas no projeto quanto à } \\
\text { redução do consumo de água para fins potáveis e para fins não potáveis, tais como o aproveitamento das águas } \\
\text { pluviais, cinzentas ou negras, levando em consideração os condicionamentos de impacto ambiental, económico e social. }\end{array}$ & 1 - Hid._Est.Prel. \\
\hline WEp1: Parâmetros de qualidade da água a aproveitar e condições de drenagem da rede pública. & 1 - Hid._Est.Prel. \\
\hline $\begin{array}{l}\text { WEp1/WEc2/WEc3: Identificação dos níveis de conforto pretendidos para os sistemas (ruídos, acessibilidade dos } \\
\text { sistemas, odores, etc.). }\end{array}$ & 1 - Hid._Est.Prel. \\
\hline $\begin{array}{l}\text { SSc5.1: Informações/registos indicando todos os elementos naturais do local (corpos de água, condições do solo, } \\
\text { ecossistemas, árvores, etc.) onde será desenvolvido o edifício. }\end{array}$ & 1 - Arq._Est.Prel. \\
\hline $\begin{array}{l}\text { SSc6.1/SSc6.2: Memorial descritivo e justificativo relevantes do Estudo Preliminar no que se refere às estratégias de } \\
\text { controle da qualidade e quantidade das águas pluviais. }\end{array}$ & 1 - Arq._Est.Prel. \\
\hline \begin{tabular}{l} 
Normas técnicas pertinentes. \\
\hline
\end{tabular}
\end{tabular}

Fonte: Produção dos autores. 
Figura 4 - Atividades técnicas a produzir na especialidade de hidráulica na fase de Anteprojeto

\begin{tabular}{|c|c|c|}
\hline Atividades/Informações técnicas a produzir & Parâmetros LEED & Normas \\
\hline 1 - Aproveitamento de águas pluviais (WEC1/WEc2) & - & - \\
\hline $\begin{array}{l}\text { - A água não potável proveniente da captação da água pode ser utilizada em para limpeza de bacias sanitárias e } \\
\text { mictórios, para combate a incêndios e outros usos onde o requisito de potabilidade não se faça necessário. }\end{array}$ & WEc1/WEc2 & NBR 5626:1998 \\
\hline $\begin{array}{l}\text { - o sistema de cobertura deve ter capacidade para drenar a máxima precipitação possivel, evitando empoçamentos ou } \\
\text { extravasamentos para o interior da edificação habitacional ou quaisquer outros locais não previstos no projeto. }\end{array}$ & WEc1/WEc2 & NBR 15575:2013 \\
\hline - O fundo do reservatório deve ficar acima do nível de água máximo do lençol freático e da cota de inundação máxima. & WEc1/WEc2 & NBR 12217:1994 \\
\hline $\begin{array}{l}\text { - O nível do lençol freático pode ser rebaixado mediante drenagem e descarga por gravidade. A drenagem deve circundar } \\
\text { o fundo do reservatório a uma distância que garanta a estabilidade da estrutura e previna riscos de contaminação }\end{array}$ & WEc1/WEc2 & NBR 12217:1994 \\
\hline $\begin{array}{l}\text { - Determinar o volume de águas pluviais aproveitáveis para posteriormente se proceder ao dimensionamento do } \\
\text { reservatório. O volume de águas pluviais pode ser determinado através da seguinte equação: } \\
\text { [ V=P*A*C*n] } \\
\text { Onde: } \\
\text { V-Volume anual, mensal ou diário de água das chuvas aproveitável; } \\
\text { P-Precipitação média anual, mensal ou diária; } \\
\text { A - Área de captação; } \\
\text { C - Coeficiente de escoamento superficial da cobertura; } \\
\text { n- Eficiência do sistema de captação, levando em conta o dispositivo de descarte de sólidos e "first flush" caso seja } \\
\text { utilizado }\end{array}$ & WEc1/WEc2 & NBR 15527:2007 \\
\hline $\begin{array}{l}\text { Determinar os caudais de cálculo para se proceder ao dimensionamento do sistema de drenagem das águas pluviais. Os } \\
\text { caudais de cálculo podem ser determinados através da seguinte formula: } \\
\text { [ Q=I*A/60] } \\
\text { Onde: } \\
\text { Q- Caudal de cálculo }(1 / \mathrm{min} .) ; \\
\text { I- Intensidade pluviométrica }\left(\mathrm{mm} / \mathrm{h}=1 / \mathrm{h}^{*} \mathrm{~m} 2\right) ; \\
\text { A-Área de contribuição }(\mathrm{m} 2) \text {. }\end{array}$ & & \\
\hline 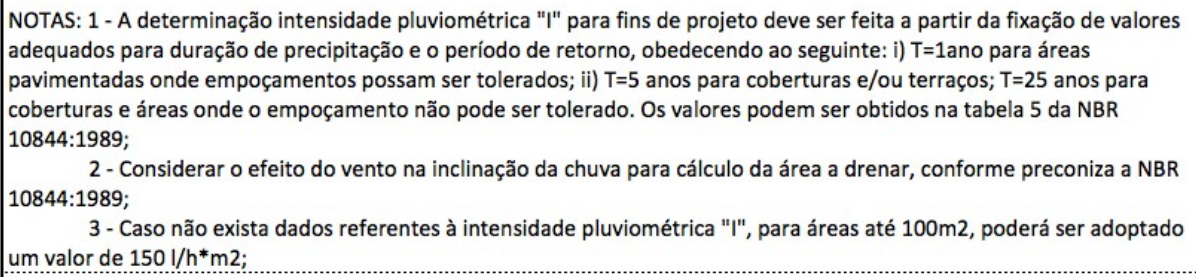 & WEc1/WEc2 & NBR 10844:1989 \\
\hline $\begin{array}{l}\text { - Realizar um pré-dimensionamento do reservatório, bem como a sua localização e principais componentes (tubagens, sistemas } \\
\text { de tratamento, etc.). }\end{array}$ & WEc1/WEc2 & NBR 15527:2007 \\
\hline - $\mathrm{T}$ & WEc1/WEc2 & NBR 15575:2013 \\
\hline
\end{tabular}

Fonte: Produção dos autores.

Figura 5 - Documentos técnicos a apresentar na especialidade de hidráulica na fase de Anteprojeto

\begin{tabular}{|c|c|c|}
\hline Saídas/Documentos técnicos a apresentar & & ID destino \\
\hline $\begin{array}{l}\text { WEp1/WEc2/WEc3: Dimensionamento aproximado relativamente ao planeamento da rede de canalização do } \\
\text { edifício, indicando o traçado, instalações hidráulicas e os tipos de drenagem previstos. }\end{array}$ & 1 - Hid._Proj.Leg. & 2 - Est._Proj.Leg. \\
\hline $\begin{array}{l}\text { WEp1/WEc2/WEc3: Memorial descritivo e justificativo relevantes do Anteprojeto no que se refere às } \\
\text { instalações hidráulicas quanto ao aproveitamento das águas pluviais, cinzentas ou negras. }\end{array}$ & 1 - Hid._Proj.Leg. & - \\
\hline WEp1/WEc2/WEc3: Volume de águas pluviais e cinzentas a aproveitar pelo(s) sistema(s). & 1 - Hid._Proj.Leg. & - \\
\hline $\begin{array}{l}\text { WEp1/WEc2/WEc3: Identificação dos níveis de conforto pretendidos para os sistemas (ruídos, acessibilidade } \\
\text { dos sistemas, odores, etc.). }\end{array}$ & 1 - Hid._Proj.Leg. & - \\
\hline
\end{tabular}

Fonte: Produção dos autores.

De forma sucinta, os processos apresentam as seguintes características:

- Cada processo é constituído por três partes. A primeira parte (Figura 3) corresponde a um conjunto de documentos que contem "informações de referência a utilizar". Estes documentos têm como objetivo auxiliar o projetista da respetiva especialidade no 
desenvolvimento de determinadas "atividades técnicas a produzir" permitindo assim incorporar no projeto soluções construtivas adequadas. Importa referir que as "atividades técnicas a produzir" (Figura 4) não é mais que um conjunto de informações/indicadores que o projetista deverá ter em consideração no desenvolvimento de determinadas soluções construtivas. A terceira e última parte (Figura 5) corresponde aos documentos técnicos que o projetista deve apresentar para validar as soluções construtivas que concebeu;

- Os documentos correspondentes às "informações técnicas a utilizar" estão devidamente identificados do processo que lhe deu origem. Por exemplo na Figura 3 verifica-se que os documentos que servem de referência ao Anteprojeto tem por base o Estudo Preliminar da especialidade de hidráulica (ID - Hid._Est.Prel.) e da arquitetura (ID - Arq._Est.Prel.). Estes documentos servirão de apoio para as atividades a desenvolver neste processo Hid._Antepr (Hidráulica Anteprojeto);

- As "informações técnicas a produzir" (figura 4), permite auxiliar o projetista a atingir os objetivos previstos nesta fase de projeto. Com efeito, cada indicador está devidamente identificado em função do parâmetro LEED-NC que se pretende atingir, bem como a referência normativa para garantir a qualidade técnica das soluções que irão ser incorporadas no projeto. Também nesta etapa cada indicador está devidamente identificado em função do parâmetro LEED-NC que se pretende atender, bem como a referência normativa para garantir a qualidade técnica das soluções e sistemas construtivos que irão ser incorporadas no projeto. Tomando a Figura 4 como exemplo, no que se refere à eficiência hídrica do edifício um dos parâmetros avaliados pelo LEED-NC é reduzir o consumo de água potável da rede pública; neste sentido uma das estratégias pode passar pelo reaproveitamento das águas pluviais; torna-se então necessário nesta etapa de Anteprojeto determinar o volume de água pluvial a aproveitar para se proceder à escolha das melhores soluções construtivas para sistema de drenagem e do reservatório. Por conseguinte, o correto dimensionamento do sistema de drenagem é especificado pela norma NBR 15.527:2007. Cada parâmetro LEED-NC a incorporar no projeto encontra-se devidamente apoiado em documentos normativos para garantir a qualidade da solução técnica que se pretende introduzir no projeto;

- Os "documentos técnicos a apresentar" (Figura 5) constituem os resultados das soluções construtivas definidas no projeto. Estes documentos técnicos irão servir de documentos de referência/entrada noutros processos, podendo ser na mesma especialidade ou em outras, caso se verifique necessário. Por exemplo, caso o projetista introduza um sistema de aproveitamento de águas pluviais, de acordo com a figura 5 , verifica-se que 0 dimensionamento do sistema de drenagem das águas pluviais tem como saídas o Projeto Legal na especialidade de hidráulica (ID Hid._Proj.Leg.) e estruturas (ID Est._Proj.Leg.), permitindo ao projetista de estruturas durante a etapa seguinte proceder ao dimensionamento que servirá de suporte aos elementos do sistema de aproveitamento de águas pluviais (tais como dimensionamento do suporte do reservatório). Analisando o mapa de processos da Figura 2 constata-se exatamente isso, ou seja, o processo que corresponde à etapa de Anteprojeto para a especialidade de instalações hidráulicas apresenta a interligação ao processo de projeto legal na especialidade de estruturas e hidráulicas.

Cada processo e de acordo com a especialidade em questão foram levados em consideração diferentes documentos normativos. As tabelas seguintes apresentam a relação entre eles. 
Quadro 1 - Normas introduzidas na especialidade de instalações hidráulicas

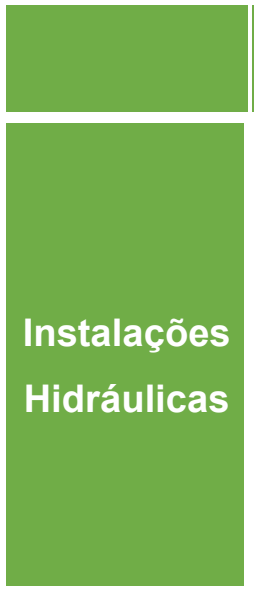

\section{Normas Aplicadas}

NBR 5626:1998 - Instalação predial de água fria

NBR 10844:1989 - Instalações prediais de águas pluviais

NBR 15527:2007 - Água da Chuva Aproveitamento de coberturas em áreas urbanas para fins não potáveis

NBR 13969:1997 - Tanques Sépticos - Unidades de Tratamento Complementar e disposição final dos efluentes líquidos

NBR 12217:1994 - Projeto de Reservatório de Distribuição de água para abastecimento público

NBR 8160:1999 - Sistemas Prediais de esgoto sanitário - projeto e execução ETA 0107 - Sistemas de Aproveitamento de Águas Pluviais em Edifícios

ETA 0905 - Sistemas de Reaproveitamento e Reciclagem de Águas Cinzenta

Fonte: Produção dos autores.

Quadro 2 - Normas introduzidas na especialidade de instalações hidráulicas

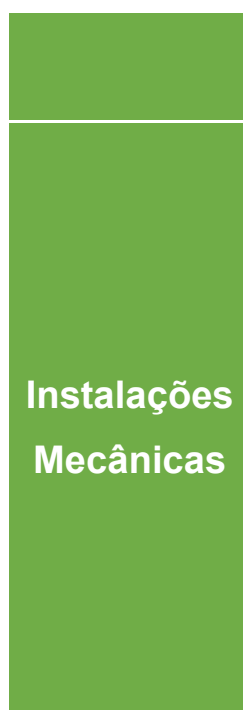

\section{Normas Aplicadas}

NBR 15575:2013 - Norma de Desempenho de Edifícios Habitacionais.

NBR 15220:2008 - Desempenho Térmico das Habitações

Deliberação Normativa COPAM $n^{\circ} 160 / 2010$

ASHRAE 62.1-2007 - Ventilation for Acceptable Indoor Air Quality

ASHRAE 90.1-2007 - Energy Standard for Buildings Except Low-Rise Residential Buildings

EN 779:2012 - Particulate Air Filters for General Ventilation - Determination of the Filtration Performance

EN 15251:2007 - Indoor environmental input parameters for design and assessment of energy performance of buildings addressing indoor air quality, thermal environment, lighting and acoustics

ASHRAE 55-2013 - Thermal Environmental Conditions for Human Occupancy ISO 7730:2005 - Ergonomics of the thermal environment, Analytical determination and interpretation of thermal comfort

Fonte: Produção dos autores. 


\section{1}

Quadro 3 - Normas introduzidas na especialidade de estruturas, arquitetura e instalações elétricas

\begin{tabular}{|c|c|}
\hline & Normas Aplicadas \\
\hline \multirow{4}{*}{ Estruturas } & NBR 10844:1989 - Instalações prediais de águas pluviais \\
\hline & NBR 15575:2013 - Norma de Desempenho de Edifícios Habitacionais \\
\hline & NBR 15220:2008 - Desempenho Térmico das Habitações \\
\hline & $\begin{array}{l}\text { NBR ISO 14021:2013 - Rótulos e declarações ambientais. Autodeclarações } \\
\text { ambientais }\end{array}$ \\
\hline \multirow[b]{2}{*}{ Arquitetura } & NBR 15575:2013 - Norma de Desempenho de Edifícios Habitacionais. \\
\hline & NBR 15220:2008 - Desempenho Térmico das Habitações \\
\hline Instalações & $\begin{array}{l}\text { NBR 15575:2013 - Norma de Desempenho de Edifícios Habitacionais. } \\
\text { IESNA RP-33 - Lighting for Exterior Environments an IESNA Recommended } \\
\text { Pratice }\end{array}$ \\
\hline Elétricas & $\begin{array}{l}\text { ASHRAE 90.1-2007 - Energy Standard for Buildings Except Low-Rise } \\
\text { Residential Buildings }\end{array}$ \\
\hline
\end{tabular}

Fonte: Produção dos autores.

\section{CONCLUSÕES}

Ao longo dos últimos anos tem-se verificado um aumento significativo do interesse pelo tema da construção sustentável, cada vez mais atual. Este trabalho, através do desenvolvimento de uma ferramenta, procurou fornecer uma perspectiva inovadora no modo como conciliar a gestão de projetos com a sustentabilidade no ambiente construído.

A ferramenta apresentada permite que, de uma forma coordenada e integrada, efetuar uma abordagem às diferentes especialidades que constituem o processo de desenvolvimento de projeto, nas suas diferentes fases de evolução, conjugando as necessidades de cada uma delas com os requisitos de sustentabilidade do sistema LEED-NC e alguns documentos normativos nacionais e internacionais pertinentes.

Relativamente a evoluções futuras, estas podem passar pela efetivação de um estudo de caso permitindo a otimização do instrumento.

\section{AGRADECIMENTOS}

À CAPES pela bolsa de pesquisa.

\section{REFERÊNCIAS}

ASSOCIAÇÃO BRASILEIRA DE NORMAS TÉCNICAS (ABNT) - NBR 13.531. Elaboração de projetos de edificações - Atividades técnicas. 1995.

BRAGANÇA, L.; VIEIRA, S. M.; ANDRADE, J. B. Early Stage Design Decisions: The Way to Achieve Sustainable Buildings at Lower Costs. The Scientific World Journal, 22 Jan. 2014.

BRAGANÇA, L.; MATEUS, R.; KOUKKARI, H. Building Sustainability Assessment.

Sustainability.2010.

CASTANHEIRA, G.; BRAGANÇA, L. The Evolution of the Sustainability Assessment Tool SBTool: From Buildings to the Built Environment. The Scientific World Journal, p. 10, 27 Jan. 2014. 
COMITÉ EUROPEU DE NORMALIZAÇÃO. EN15643-1: Sustainability of Construction Works. Sustainability Assessment of Buildings. Part 1: General Framework, 2010.

COMITÉ EUROPEU DE NORMALIZAÇÃO. EN15643-2: Sustainability of Construction Works. Assessment of Buildings - Part: Framework for the Assessment of Environmental Performance. 2011.

COMITÉ EUROPEU DE NORMALIZAÇÃO. EN15643-3: Sustainability of Construction Works. Assessment of Buildings e Part 3: Framework for the Assessment of Social performance, 2012.

COMITÉ EUROPEU DE NORMALIZAÇÃO. EN15643-4: Sustainability of Construction Works. Assessment of Buildings - Part 4: Framework for the Assessment of Economic Performance, 2012.

DEANE, M. The Builder's Role in Delivering Sustainable Tall Buildings. The Structural Design of Tall and Special Buildings, p. 869-880, 2008.

DING, G. K. Sustainable construction - the role of environmental assessment tools. Jornal of environmental Management, p. 451-464, 2008.

EN15978. (2011). Sustainability of construction works - Assessment of environmental performance of buildings - Calculation method.

FERREIRA, J.; PINHEIRO, M. D.; BRITO, J. D. Portuguese sustainable construction assessment tools benchmarked with BREEAM and LEED: An energy analysis. Energy and Buildings, p. 451-463, 08 Nov. 2013.

KIBERT, C. J. Sustainable Construction: Green Building Design and Delivery. New Jersey: John Wiley \& Sons, 2013.

KOHLER, N.; MOFFATT, S. Life-cycle analysis of the built environment. United Nations Environment Programme UNEP Industry and Environment, p.17-21, 09 Abr. 2003.

LEED. Leadership in Energy and Environmental Design for new construction and major renovations with alternative compliance paths for projects outside the U.S. USGBC, 2009.

MANENT, C. S.; KORKMAZ, S.; KLOTZ, L.E.; RILEY, D. R. A Design Process Evaluation Method for Sustainable Buildings. Architectural Engineering and Design Management, p. 6274, 2009.

MATEUS, R. Avaliação da Sustentabilidade da Construção. Tese de Douturado. Universidade do Minho, 2009.

PEREIRA, T. D. Gestão de projeto e contratação de empreitadas de obras. Coimbra: Impresa da Universidade de Coimbra, 2014.

REKOLA, M.; MÄKELÄINEN, T.; HÄKKINEN T. The Role of design management in the sustainable building process. Architectural Engineering and Design Management, p. 7889, 2012.

SON, H.; KIM, C.; CHONG, W. K.; CHOU, J.-S. Implementing Sustainable Development in the Construction Industry: Constructors' Perspectives in the US and Korea. Sustainable Development, 2009.

ZUO, J., \& ZHAO, Z.-Y. Green building research - current status and future agenda: A review. Renewable and Sustainable Energy Reviews, p. 271-281, 2013. 